\title{
Reduction of flexible joint manipulator mathematical model
}

\author{
O.V. Vidilina ${ }^{1}$, N.V. Voropaeva ${ }^{1}$ \\ ${ }^{1}$ Samara National Research University, 34, Moskovskoye shosse, 443086, Samara, Russia
}

\begin{abstract}
The singularly perturbed differential systems which describe the dynamics of the manipulator with flexible joints are investigated under the condition of weak dissipation. The method of integral manifolds is used to construct the reduced model of robot. Integral manifolds may be constructed as an asymptotic power series. The simplified model is used to construct the control law for the robot with two flexible joints.
\end{abstract}

Keywords: mathematical model; integral manifolds; reduction; asymptotic methods

\section{Introduction}

The dynamic and control problems for robotic systems are connected with difficulties caused by high dimensions of models and availability of several time scales. Thereby the reduction problem ( the problem of the construction the lower order corrected models) is topical.

We investigate the model of $n$-links robot-manipulator with flexible joints where dissipation is small. The dynamics of such manipulators is described by quasi-oscillating singularly perturbed differential systems, which contain small parameter at the leading derivative. The conditions ensuring the possibility of using classical asymptotic methods are described in the wellknown Tikhonov's theorem. The main of them is the asymptotic stability of the so-called boundary layer system. For investigated class of systems this condition of Tikhonov's theorem is not satisfied.

One of the approaches, which allows to reduce the complex multirate dynamic systems, is based on the theory of integral manifolds [1-14]. The conditions of the existence of an attractive slow integral manifold are investigated. This makes it possible to use the slow subsystem, which describes the motion on the manifold, as the simplified model of the manipulator. Similar questions for other classes of quasi-oscillating systems are studied in [10-13].

We consider the dynamic model of $n$-links robot with flexible joints Fig. 1.

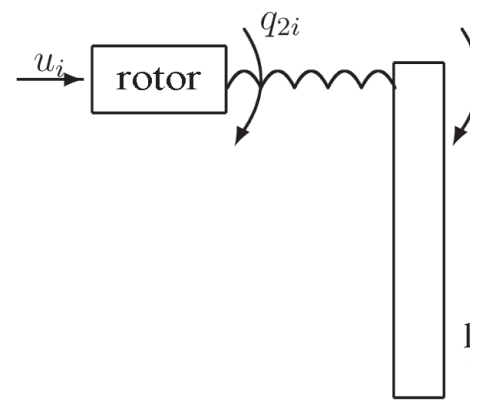

Fig. 1. The link of the manipulator.

The dynamics of manipulator is described by the system [15 - 18]

$$
\begin{array}{r}
D\left(q_{1}\right) \ddot{q}_{1}+c\left(q_{1}, \dot{q}_{1}\right)+K\left(q_{1}-q_{2}\right)+B\left(\dot{q}_{1}-\dot{q}_{2}\right)=0, \\
J \ddot{q}_{2}-K\left(q_{1}-q_{2}\right)-B\left(\dot{q}_{1}-\dot{q}_{2}\right)=u,
\end{array}
$$

where the coordinates of vectors $q_{1} \in R^{n}$ and $q_{2} \in R^{n}$ are the angles which characterize links and rotors positions respectively, $D\left(q_{1}\right)$ is inertia matrix due to the links, $J$ is diagonal inertia matrix of drive rotors, vector $c\left(q_{1}, \dot{q}_{1}\right)$ is determined by coriolis, centrifugal and gravitation components. The flexibility of joint is represented by torsion spring with sufficiently large elastic coefficient. Let $K=k \operatorname{diag}\left(\widetilde{K}_{1}, \ldots, \widetilde{K}_{n}\right)$ be the matrix of elastic coefficients, $B=\operatorname{diag}\left(B_{1}, \ldots, B_{n}\right)$ be the matrix of damping ratios, $u$ be the unit torque.

Let $\mu=1 / k$ be a small positive parameter. Note that the more hard restriction on the matrix $B$ was imposed in [15-18]. It was supposed that $B_{j}=\widetilde{B}_{j} / \mu$, or $B_{j}=\widetilde{B}_{j} / \sqrt{\mu}$. In fact, it was assumed that there is a sufficiently high dissipation. Such assumption guarantees the fulfillment of Tichonov's theorem condition about the asymptotic stability of the boundary layer system. Let us suppose that $B_{j}=O(1)$. Then the main condition of this theorem is not fulfilled. 


\section{Reduction of the model}

Putting $q=q_{1}, z=k\left(q_{1}-q_{2}\right)$ gives us the following system

$$
\begin{aligned}
\ddot{q} & =a_{1}(q, \dot{q})+A_{1}(q) z+\mu A_{3}(q) \dot{z}, \\
\mu \ddot{z} & =a_{2}(q, \dot{q})+A_{2}(q) z+\mu A_{4}(q) \dot{z}+M_{2} u,
\end{aligned}
$$

where

$$
\begin{array}{ll}
a_{1}(q, \dot{q})=a_{2}(q, \dot{q})=-D^{-1}(q) c(q, \dot{q}), \quad A_{1}(q)=-D^{-1}(q) \widetilde{K} \\
A_{2}(q)=-\left(D^{-1}(q)+J^{-1}\right) \widetilde{K}, \quad A_{3}(q)=-D^{-1}(q) B, \\
A_{4}(q)=-\left(D^{-1}(q)+J^{-1}\right) B, \quad M_{2}=-J^{-1} .
\end{array}
$$

Using the coordinates $x_{1}=q, x_{2}=\dot{q}, y_{1}=z, y_{2}=\dot{z}$ we can rewrite system (2) to the form

$$
\begin{aligned}
\dot{x}_{1} & =x_{2}, \\
\dot{x}_{2} & =a_{1}(x)+A_{1}\left(x_{1}\right) y_{1}+\mu A_{3}\left(x_{1}\right) y_{2}, \\
\mu \dot{y}_{1} & =\mu y_{2}, \\
\mu \dot{y}_{2} & =a_{2}(x)+A_{2}\left(x_{1}\right) y_{1}+\mu A_{4}\left(x_{1}\right) y_{2}+M_{2} u(t, x, \mu .
\end{aligned}
$$

We obtained [14] the conditions for the existence of attractive slow integral manifold of system (3)

$$
y=h(t, x, \mu)
$$

where $x=\left(\begin{array}{l}x_{1} \\ x_{2}\end{array}\right), \quad y=\left(\begin{array}{l}y_{1} \\ y_{2}\end{array}\right), \quad h(t, x, \mu)=\left(\begin{array}{l}h_{1}(t, x, \mu) \\ h_{2}(t, x, \mu)\end{array}\right)$.

Let function $u(t, x, \mu)$ is represented in the form $u(t, x, \mu)=u_{0}(t, x)+\mu u_{1}(t, x)+\ldots$

Integral manifold (4) may be constructed as an asymptotic power series of the small parameter $\mu$

$$
y_{i}=h_{i}^{(0)}(t, x)+\mu h_{i}^{(1)}(t, x)+\mu^{2} h_{i}^{(2)}(t, x)+\ldots, \quad i=1,2
$$

with any degree of accuracy. Substituting (5) to the equations

$$
\begin{aligned}
& \frac{\partial h_{1}}{\partial t}+\frac{\partial h_{1}}{\partial x_{1}} x_{2}+\frac{\partial h_{1}}{\partial x_{2}}\left(a_{1}(x)+A_{1}\left(x_{1}\right) h_{1}+\mu A_{3}\left(x_{1}\right) h_{2}\right)=h_{2}, \\
& \mu\left(\frac{\partial h_{2}}{\partial t}+\frac{\partial h_{2}}{\partial x_{1}} x_{2}+\frac{\partial h_{2}}{\partial x_{2}}\left(a_{1}(x)+A_{1}\left(x_{1}\right) h_{1}+\mu A_{3}\left(x_{1}\right) h_{2}\right)=\right. \\
& =a_{2}(x)+A_{2}\left(x_{1}\right) h_{1}+\mu A_{4}\left(x_{1}\right) h_{2}+M_{2} u(t, x, \mu), \\
& h_{i}=h_{i}(t, x, \mu)
\end{aligned}
$$

and equating the coefficients at the same powers of $\mu$ we can get $h_{i}^{(j)}=h_{i}^{(j)}(t, x)$ for any $j$. In particular

$$
\begin{aligned}
& h_{1}^{(0)}=-A_{2}^{-1}\left(x_{1}\right)\left[a_{2}(x)+M_{2} u_{0}(t, x)\right], \quad h_{2}^{(0)}=\frac{\partial h_{1}^{(0)}}{\partial t}+\frac{\partial h_{1}^{(0)}}{\partial x_{1}} x_{2}+\frac{\partial h_{1}^{(0)}}{\partial x_{2}}\left[a_{1}(x)+A_{1}\left(x_{1}\right) h_{1}^{(0)}\right], \\
& h_{1}^{(1)}=A_{2}^{-1}\left(x_{1}\right)\left[\frac{\partial h_{2}^{(0)}}{\partial t}+\frac{\partial h_{2}^{(0)}}{\partial x_{1}} x_{2}+\frac{\partial h_{2}^{(0)}}{\partial x_{2}}\left[a_{1}(x)+A_{1}\left(x_{1}\right) h_{1}^{(0)}\right]-A_{4}\left(x_{1}\right) h_{2}^{(0)}-M_{2} u_{1}(t, x)\right], \\
& h_{2}^{(1)}=\frac{\partial h_{1}^{(1)}}{\partial t}+\frac{\partial h_{1}^{(1)}}{\partial x_{1}} x_{2}+\frac{\partial h_{1}^{(1)}}{\partial x_{2}}\left[a_{1}(x)+A_{1}\left(x_{1}\right) h_{1}^{(0)}\right]+\frac{\partial h_{1}^{(0)}}{\partial x_{2}}\left[A_{1}\left(x_{1}\right) h_{1}^{(1)}+A_{3}\left(x_{1}\right) h_{2}^{(0)}\right] .
\end{aligned}
$$

For $h_{i}^{(j)}, i=1,2$ from (6) we have

$$
\begin{aligned}
h_{1}^{(j)} & =A_{2}^{-1}\left(x_{1}\right)\left[M_{2} u_{j}(t, x)-A_{4}\left(x_{1}\right) h_{2}^{(j-1)}-\frac{\partial h_{2}^{(j-1)}}{\partial t}-\frac{\partial h_{2}^{(j-1)}}{\partial x_{1}} x_{2}-\frac{\partial h_{2}^{(j-1)}}{\partial x_{2}}\left[a_{1}(x)+A_{1}\left(x_{1}\right) h_{1}^{(0)}\right]-\right. \\
& \left.-\sum_{s=0}^{j-2} \frac{\partial h_{2}^{(s)}}{\partial x_{2}}\left[A_{1}\left(x_{1}\right) h_{1}^{(j-s-1)}+A_{3}\left(x_{1}\right) h_{2}^{(j-s-2)}\right]\right], \\
h_{2}^{(j)} & =\frac{\partial h_{1}^{(j)}}{\partial t}+\frac{\partial h_{1}^{(j)}}{\partial x_{1}} x_{2}+\frac{\partial h_{1}^{(j)}}{\partial x_{2}}\left[a_{1}(x)+A_{1}\left(x_{1}\right) h_{1}^{(0)}\right]+\sum_{s=0}^{j-1} \frac{\partial h_{1}^{(s)}}{\partial x_{2}}\left[A_{1}\left(x_{1}\right) h_{1}^{(j-s)}+A_{3}\left(x_{1}\right) h_{2}^{(j-1-s)}\right] .
\end{aligned}
$$


The system, which describes the motion on the slow integral manifold, is

$$
\begin{aligned}
& \dot{x_{1}}=x_{2}, \\
& \dot{x_{2}}=a_{1}(x)+A_{1}\left(x_{1}\right) h_{1}(t, x, \mu)+\mu A_{3}\left(x_{1}\right) h_{2}(t, x, \mu) .
\end{aligned}
$$

The dimension of this system is half of the dimension of the initial system. The slow subsystem has not fast variables, but nonetheless reliably describes the behavior of full system near the slow integral manifold. This allows to use it as a simplified model of flexible joints robot. The proposed approach to construct the reduced model is used in [11 - 13] to solve the problems of control and estimation for robot with one flexible joint.

\section{Example}

Let us consider the control problem for the robot with two flexible joints. The dynamics of manipulator is described by system (1), where [18]

$$
\begin{aligned}
& D\left(q_{1}\right)=\left(\begin{array}{cc}
\theta_{1}+\theta_{2}+2 \theta_{3} \cos \varphi_{2} & \theta_{2}+\theta_{3} \cos \varphi_{2} \\
\theta_{2}+\theta_{3} \cos \varphi_{2} & \theta_{2}
\end{array}\right), \quad q_{1}=\left(\begin{array}{c}
\varphi_{1} \\
\varphi_{2}
\end{array}\right), \quad q_{2}=\left(\begin{array}{l}
\psi_{1} \\
\psi_{2}
\end{array}\right), \\
& \theta_{1}=m_{1} l_{c_{1}}^{2}+m_{2} l_{1}^{2}+I_{1}, \quad \theta_{2}=m_{2} l_{c_{2}}^{2}+I_{2}, \quad \theta_{3}=m_{2} l_{1} l_{c_{2}}, \quad \theta_{4}=m_{1} l_{c_{1}}, \quad \theta_{5}=m_{2} l_{1}, \quad \theta_{6}=m_{2} l_{c_{2}}, \\
& c\left(q_{1}, \dot{q}_{1}\right)=\theta_{3} \sin \varphi_{2}\left(\begin{array}{c}
-2 \dot{\varphi}_{1} \dot{\varphi}_{2}-\dot{\varphi}_{2}^{2} \\
\dot{\varphi}_{1}^{2}
\end{array}\right)+\left(\begin{array}{c}
\left(\theta_{4}+\theta_{5}\right) g \cos \varphi_{1}+\theta_{6} g \cos \left(\varphi_{1}+\varphi_{2}\right) \\
\theta_{6} g \cos \left(\varphi_{1}+\varphi_{2}\right)
\end{array}\right), \\
& J=\left(\begin{array}{cc}
J_{1} & 0 \\
0 & J_{2}
\end{array}\right), \quad K=\left(\begin{array}{cc}
k & 0 \\
0 & k
\end{array}\right), \quad B=\left(\begin{array}{ll}
b & 0 \\
0 & b
\end{array}\right) .
\end{aligned}
$$

Using the coordinates $x_{1}=q, x_{2}=\dot{q}, y_{1}=z, y_{2}=\dot{z}$ we rewrite system (2) to the form (3), where

$$
\begin{aligned}
& x=\left(\begin{array}{c}
x_{1} \\
x_{2}
\end{array}\right), \quad x_{1}=\left(\begin{array}{c}
x_{1}^{(1)} \\
x_{2}^{(1)}
\end{array}\right)=\left(\begin{array}{c}
\varphi_{1} \\
\varphi_{2}
\end{array}\right), \quad x_{2}=\left(\begin{array}{c}
x_{1}^{(2)} \\
x_{2}^{(2)}
\end{array}\right)=\left(\begin{array}{c}
\dot{\varphi}_{1} \\
\dot{\varphi}_{2}
\end{array}\right), \quad y=\left(\begin{array}{c}
y_{1} \\
y_{2}
\end{array}\right), \\
& a_{1}(x)=a_{2}(x)=\frac{\left(-\theta_{3} \sin x_{2}^{(1)}\left(2 x_{1}^{(2)}+x_{2}^{(2)}\right) x_{2}^{(2)}+\theta_{4} g \cos x_{1}^{(1)}+\theta_{6} g \cos \left(x_{1}^{(1)}+x_{2}^{(1)}\right)\right)}{\Delta}\left(\begin{array}{c}
-\theta_{2} \\
\left(\theta_{2}+\theta_{3} \cos x_{2}^{(1)}\right)
\end{array}\right)+ \\
& +\frac{\left(\theta_{3} \sin x_{2}^{(1)}\left(x_{1}^{(2)}\right)^{2}+\theta_{6} g \cos \left(x_{1}^{(1)}+x_{2}^{(1)}\right)\right)}{\Delta}\left(\begin{array}{c}
\left(\theta_{2}+\theta_{3} \cos x_{2}^{(1)}\right) \\
-\left(\theta_{1}+\theta_{2}+2 \theta_{3} \cos x_{2}^{(1)}\right)
\end{array}\right), \quad \Delta=\theta_{1} \theta_{2}-\theta_{3}^{2} \cos ^{2} x_{2}^{(1)}, \\
& A_{1}(x)=\frac{1}{\Delta}\left(\begin{array}{cc}
-\theta_{2} & \theta_{2}+\theta_{3} \cos x_{2}^{(1)} \\
\theta_{2}+\theta_{3} \cos x_{2}^{(1)} & -\left(\theta_{1}+\theta_{2}+2 \theta_{3} \cos x_{2}^{(1)}\right)
\end{array}\right) \\
& A_{2}(x)=\frac{k}{\Delta J_{1} J_{2}}\left(\begin{array}{cc}
-J_{2}\left(\theta_{2} J_{1}+\Delta\right) & J_{1} J_{2}\left(\theta_{2}+\theta_{3} \cos x_{2}^{(1)}\right) \\
J_{1} J_{2}\left(\theta_{2}+\theta_{3} \cos x_{2}^{(1)}\right) & J_{1}\left(J_{2}\left(\theta_{1}+\theta_{2}+2 \theta_{3} \cos x_{2}^{(1)}\right)+\Delta\right)
\end{array}\right) \\
& A_{3}(x)=\frac{b}{\Delta}\left(\begin{array}{cc}
-\theta_{2} & J_{1} J_{2}\left(\theta_{2}+\theta_{3} \cos x_{2}^{(1)}\right) \\
\left(\theta_{2}+\theta_{3} \cos x_{2}^{(1)}\right) & -\left(\theta_{1}+\theta_{2}+2 \theta_{3} \cos x_{2}^{(1)}\right)
\end{array}\right)
\end{aligned}
$$$$
A_{4}(x)=\frac{b}{\Delta J_{1} J_{2}}\left(\begin{array}{cc}
-J_{2}\left(\theta_{2} J_{1}+\Delta\right) & J_{1} J_{2}\left(\theta_{2}+\theta_{3} \cos x_{2}^{(1)}\right) \\
J_{1} J_{2}\left(\theta_{2}+\theta_{3} \cos \left(x_{2}^{(1)}\right)\right) & J_{1}\left(J_{2}\left(\theta_{1}+\theta_{2}+2 \theta_{3} \cos x_{2}^{(1)}\right)+\Delta\right)
\end{array}\right), \quad M_{2}=\left(\begin{array}{cc}
-\frac{1}{J_{1}} & 0 \\
0 & -\frac{1}{J_{2}}
\end{array}\right) .
$$

The slow integral manifold (4) takes the form (5), where coefficients $h_{j, k}^{(i)}$ are obtained from (7)by using the computer algebra system Maple. In particular

$$
\begin{aligned}
h_{1,1}^{(0)} & =-\frac{1}{k S}\left(u_{1}^{(0)}\left(\theta_{1} \theta_{2}+J_{2} \theta_{1}+J_{2} \theta_{2}-\theta_{3}^{2}\left(\cos x_{2}^{(1)}\right)^{2}+2 J_{2} \theta_{3} \cos x_{2}^{(1)}\right)+u_{2}^{(0)} J_{1}\left(\theta_{2}+\theta_{3} \cos x_{2}^{(1)}\right)-\right. \\
& -J_{1} \theta_{3}^{2}\left(x_{1}^{(2)}\right)^{2} \cos x_{2}^{(1)} \sin x_{2}^{(1)}-J_{1} \theta_{3} \sin x_{2}^{(1)}\left(\theta_{2}\left(x_{1}^{(2)}+x_{2}^{(2)}\right)^{2}+J_{2} x_{2}^{(2)}\left(2 x_{1}^{(2)}+x_{2}^{(2)}\right)\right)+ \\
& \left.+J_{1} g\left(\theta_{4}+\theta_{5}\right) \cos x_{1}^{(1)}\left(\theta_{2}+J_{2}\right)+\cos \left(x_{1}^{(1)}+x_{2}^{(1)}\right) J_{1} g \theta_{6}\left(J_{2}-\theta_{3} \cos x_{2}^{(1)}\right)\right), \\
h_{1,2}^{(0)} & =-\frac{1}{k S}\left(u_{1}^{(0)} J_{2}\left(\theta_{2}+\theta_{3} \cos x_{2}^{(1)}\right)+u_{2}^{(0)}\left(\theta_{1} \theta_{2}+J_{1} \theta_{2}-\theta_{3}^{2}\left(\cos x_{2}^{(1)}\right)^{2}\right)-J_{2} g\left(\theta_{4}+\theta_{5}\right) \cos x_{1}^{(1)}\left(\theta_{2}+\theta_{3} \cos x_{2}^{(1)}\right)+\right.
\end{aligned}
$$




$$
\begin{aligned}
+ & J_{2} g \theta_{6} \cos \left(x_{1}^{(1)}+x_{2}^{(1)}\right)\left(\theta_{1}+J_{1}+\theta_{3} \cos x_{2}^{(1)}\right)+J_{2} \theta_{3}^{2} \cos x_{2}^{(1)} \sin x_{2}^{(1)}\left(2 x_{1}^{(2)} x_{2}^{(2)}+\left(x_{2}^{(2)}\right)^{2}+2\left(x_{1}^{(2)}\right)^{2}\right)+ \\
& \left.+J_{2} \theta_{3} \sin x_{2}^{(1)}\left(\left(\theta_{1}+\theta_{2}\right)\left(x_{1}^{(2)}\right)^{2}+2 \theta_{2} x_{1}^{(2)} x_{2}^{(2)}+\theta_{2}\left(x_{2}^{(2)}\right)^{2}+J_{1}\left(x_{1}^{(2)}\right)^{2}\right)\right), \\
S & =\left(J_{2} \theta_{1}+\theta_{1} \theta_{2}+J_{1} \theta_{2}+J_{2} \theta_{2}+J_{1} J_{2}-\theta_{3}^{2}\left(\cos x_{2}^{(1)}\right)^{2}+2 J_{2} \theta_{3} \cos x_{1}^{(1)}\right) .
\end{aligned}
$$

The reduced system (8) takes the form

$$
\begin{aligned}
\dot{x}_{1}^{(1)} & =x_{1}^{(2)}, \quad \dot{x}_{2}^{(1)}=x_{2}^{(2)}, \\
\dot{x}_{1}^{(2)} & =\frac{1}{S}\left(u_{1}^{(0)}\left(\theta_{2}+J_{2}\right)-u_{2}^{(0)}\left(\theta_{2}+\theta_{3} \cos x_{2}^{(1)}\right)-g\left(\theta_{4}+\theta_{5}\right) \cos x_{1}^{(1)}\left(\theta_{2}+J_{2}\right)+\theta_{3}^{2} \cos x_{2}^{(1)} \sin x_{2}^{(1)}\left(x_{1}^{(2)}\right)^{2}+\right. \\
& +\theta_{3} \sin x_{2}^{(1)}\left(\theta_{2}\left(x_{1}^{(2)}+x_{2}^{(2)}\right)^{2}+J_{2} x_{2}^{(2)}\left(x_{2}^{(2)}+2 x_{1}^{(2)}\right)\right)+g \theta_{6} \cos \left(x_{1}^{(1)}+x_{2}^{(1)}\right)\left(\theta_{3} \cos x_{2}^{(1)}-J_{2}\right)+O(\mu), \\
\dot{x}_{2}^{(2)} & =\frac{1}{S}\left(u_{1}^{(0)}\left(\theta_{2}+\theta_{3} \cos x_{2}^{(1)}\right)-u_{2}^{(0)}\left(\theta_{1}+\theta_{2}+2 \theta_{3} \cos x_{2}^{(1)}+J_{1}\right)-\cos x_{1}^{(1)}\left(\theta_{4}+\theta_{5}\right) g\left(\theta_{2}+\theta_{3} \cos x_{2}^{(1)}\right)+\right. \\
& +\theta_{3} \sin x_{2}^{(1)}\left(\theta_{2} x_{2}^{(2)}\left(x_{2}^{(2)}+2 x_{1}^{(2)}\right)+\left(x_{1}^{(2)}\right)^{2}\left(\theta_{1}+\theta_{2}+J_{1}\right)\right)+\theta_{3}^{2} \cos x_{2}^{(1)} \sin x_{2}^{(1)}\left(x_{1}^{(2)}+x_{2}^{(2)}\right)^{2}+ \\
& \left.+g \theta_{6} \cos \left(x_{1}^{(1)}+x_{2}^{(1)}\right)\left(\theta_{3} \cos x_{2}^{(1)}+J_{1}+\theta_{1}\right)\right)+O(\mu),
\end{aligned}
$$

We omitted here the terms containing $\mu$ because of their bulkiness.

We construct the control law to move both links of manipulator to the fixed stable positions. We choose the control law based on the reduced system in accordance with the concept of linearizing feedback.

The Fig. 1 and Fig. 2 demonstrate the dynamics of the angles which characterize links positions with the control law formed by reduced system for the following parameters

$$
m_{1}=10, m_{2}=5, l_{1}=1, l_{2}=1, l_{c_{1}}=0.5, l_{c_{2}}=0.5, J_{1}=1, J_{2}=1, \widetilde{K}_{1}=1, \mu=0.01, \widetilde{K}_{2}=1 .
$$

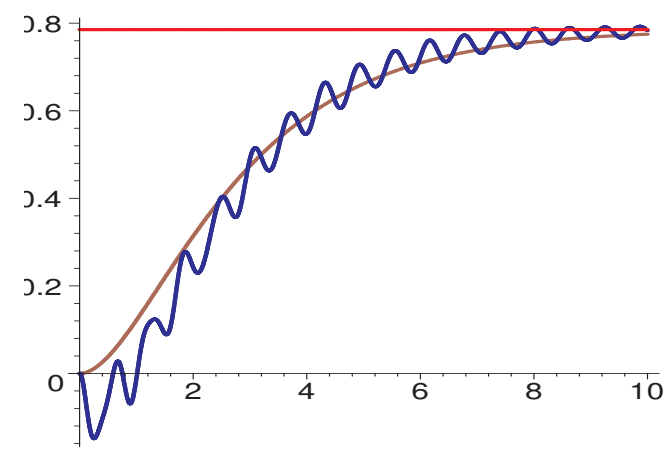

Fig. 1. The first link angle $\varphi_{1}(t)$.

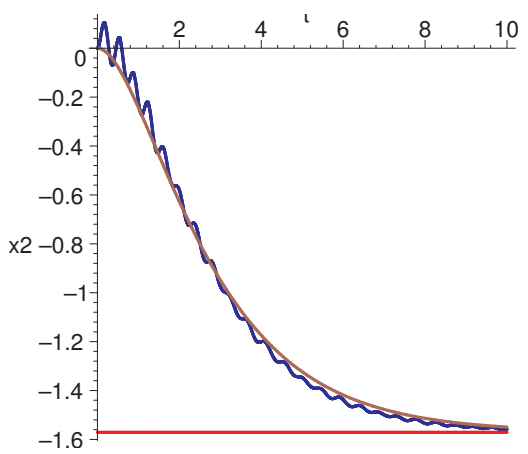

Fig. 2. The second link angle $\varphi_{2}(t)$.

It can be seen that the trajectories of initial system, which is characterized by damped high-frequency oscillations tend to the trajectories of reduced system, and those tend to the required fixed positions.

\section{Conclusion}

The application of the integral manifolds method allows us to reduce the dimension and simplify the problem of the control law construction.

\section{Acknowledgements}

The research has been supported by the Russian Foundation for Basic Research and Government of the Samara region(grant 16-41-630524).

\section{References}

[1] Sobolev VA. Integral manifolds and decomposition of singularly perturbed systems. Syst. \& Control Lett. 1984; 5: 169-279.

[2] Voropaeva NV, Sobolev VA. Geometric decomposition of singularly perturbed systems. Fizmatlit: Moscow, 2009. [in Russian]

[3] Shchepakina E, Sobolev V, Mortell MP. Singular Perturbations: Introduction to System Order Reduction Methods with Applications. In: Springer Lecture Notes in Mathematics, Cham: Springer International Publishing, 2014.

[4] Voropaeva NV, Sobolev VA. Decomposition of a linear-quadratic optimal control problem with fast and slow variables. Automation and Remote Control 2006; 67(8): 1185-1193.

[5] Voropaeva NV. Decomposition of problems of optimal control and estimation for discrete systems with fast and slow variables. Automation and Remote Control 2008; 69(6): 920-928. 
[6] Sobolev VA. Singular perturbations in linearly quadratic optimal control problems. Automation and Remote Control 1991; 52(2): 180-189.

[7] Vidilina OV, Voropaeva NV. The construction of the observers for dynamic systems with fast and slow variables. CEUR Workshop Proceedings, 2016; 1638: 750-758.

[8] Smetannikova EN, Sobolev VA. Regularization of cheap periodic control problems. Automation and Remote Control 2005; 66(6): 903-916.

[9] Strygin VV, Sobolev VA. Effect of geometric and kinetic parameters and energy dissipation on orientation stability of satellites with double spin. Cosmic Research 1976; 14(3): 366-371.

[10] Strygin VV, Sobolev VA. Separation of motions by the method of integral manifolds. Nauka: Moscow, 1988. [in Russian]

[11] Osintsev MS, Sobolev VA. Dimensionality Reduction in Optimal Control and Estimation Problems for Systems of Solid Bodies with Low Dissipation. Automation and Remote Control 2013; 74(8): 121-137.

[12] Mortell MP, O’Malley R, Pokrovskii A, Sobolev V. Singular Perturbation and Hysteresis. SIAM: Philadelphia, 2005.

[13] Aksenova NK, Sobolev VA. Control of a one rigit-link manipulator in the case of nonsmuth trajectory. CEUR Workshop Proceedings 2016; $1638: 493-497$.

[14] Vidilina OV, Voropaeva NV. Reduction of mathematical model of robot with elastic joints. Vestnik SamGU. Estestvennonauchnaya seriya. 2014; 3(114): 16-29.[in Russian]

[15] Spong MW. Modeling and control of elastic joint robots. Journal of Dynamic Systems, Measurement and Control 1987; 109: 310-319.

[16] Spong MW, Khorasani K, Kokotovic PV. An integral manifold approach to feedback control of flexible joint robots. IEEE Journal of Robotics and Automation 1987; 3(4): 291-301.

[17] Moberg S. On modeling and control of flexible manipulators. Linkoping University: Linkoping, 2007.

[18] Spong MW. On the robust control of robot manipulators. IEEE Trans. Automatic Control 1992; 37(11): 1782-1786. 